\title{
Opioid medicines management in primary care settings: a scoping review of quantitative studies of pharmacists' activities
}

\author{
Margaret Jordan ${ }^{1}$, Asam Latif ${ }^{2}$, Judy Mullan ${ }^{3}$, and Timothy Chen ${ }^{4}$ \\ ${ }^{1}$ The University of Sydney \\ ${ }^{2}$ University of Nottingham \\ ${ }^{3}$ University of Wollongong Faculty of Science Medicine and Health \\ ${ }^{4}$ University of Sydney
}

January 23, 2021

\begin{abstract}
Aim To undertake a scoping review of pharmacists' activities in opioid medicines management in primary care settings, including those developed or led by pharmacists, or in which pharmacists were members of broader multi-disciplinary teams; and to collate the activities, models of care and settings, and reported outcomes. Methods The bibliographic databases MEDLINE, EMBASE, International Pharmaceutical Abstracts, CINAHL, SCOPUS and Web of Science were searched. Studies with quantitative evaluation and published in English were eligible. Participants were patients with any pain category or an opioid use disorder, and healthcare providers. Studies originating in hospitals or involving supply functions were not included. Screening of literature and data charting of results were undertaken by two researchers. Results The 47 studies included in the scooping review occurred in primary care settings collated into four categories: general practice or primary care clinics; healthcare organisations; community pharmacies and outreach services. Studies were primarily of opioid use in chronic, non-cancer pain. Other indications were opioid use disorder, cancer and dental pain. Pharmacist activities targeted risk-mitigation, patient and provider education and broader, strategic approaches. Patient-related outcomes included reduced opioid load, improved functionality and symptom management, enhanced access to services and medication-assisted treatments, and engagement in risk-mitigation strategies. Behaviour change of providers was demonstrated. Conclusion The review has identified the significant contribution that pharmacists working in primary care settings can make to minimise harm from opioids. Strategies implemented in isolation have the potential to further reduce adverse clinical outcomes with greater collaboration and coordination, such as opioid stewardship.
\end{abstract}

\section{Hosted file}

JordanM_BJCP_Opioids primary care_301220.pdf available at https://authorea.com/users/391274/ articles/505437-opioid-medicines-management-in-primary-care-settings-a-scoping-reviewof-quantitative-studies-of-pharmacists-activities

\section{Hosted file}

JordanM_ Figure 1_PRISMA flow diagram.pdf available at https://authorea.com/users/391274/ articles/505437-opioid-medicines-management-in-primary-care-settings-a-scoping-reviewof-quantitative-studies-of-pharmacists-activities 